\title{
Chemistry's year
}

\author{
The United Nations has proclaimed 2011 to be the International Year of Chemistry. Under this banner, \\ chemists should seize the opportunity to highlight the rich history and successes of our subject to a much \\ broader audience - and explain how it can help to solve the global challenges we face today and in the future.
}

Many subjects have been the basis of 'International Year' designations by the United Nations, including peace (1986), literacy (1994), physics (2005) and even the humble potato (2008). Now, 2011 is chemistry's year, sharing the limelight with — amongst others - forests ${ }^{1}$. Hopefully chemists will be able to see the wood for the trees and take advantage of the increased profile of chemistry this year to not only celebrate specific past glories, but also to champion its role in addressing critical challenges in modern society. These global issues include sustainable energy, climate change, and the provision of clean food and water - as prominently featured in the United Nations' Resolution $^{2}$ and also on the International Year of Chemistry (IYC) website . $^{3}$

One historical aspect being brought to the fore is that 2011 is the one-hundredth anniversary of the award of the Nobel Prize in Chemistry to Marie Curie for the discovery and study of polonium and radium. Of course, this was not Curie's first Nobel Prize, having shared the physics prize in 1903 - with her husband Pierre Curie, and Henri Becquerel - for work on radioactivity. To date, Marie Curie is one of a select band of remarkable individuals who have received two Nobel Prizes, the others being Linus Pauling (Chemistry 1954, Peace 1962), John Bardeen (Physics 1956, 1972) and Fred Sanger (Chemistry 1958, 1980).

Marie Curie's scientific achievements are all the more extraordinary because they are set in the context of a very male-dominated era. Undoubtedly there are now fewer barriers and less bias faced by women in science compared with Curie's day, but nevertheless, since the inception of the Nobel Prizes in 1901, only one other woman has been awarded the physics prize and only three other women have received the chemistry prize (one of whom is Marie Curie's daughter, Irène Joliot-Curie). So although Marie Curie's contributions to chemistry should be celebrated in their own right, it is also a chance to reflect on how far gender equality has come in science - and how much further it needs to go to ensure a level playing field for women and men.

In addition to marking this significant anniversary, the IYC prospectus ${ }^{4}$ outlines a number of goals for the activities planned in 2011. The theme of broader engagement and outreach run very strongly through these and the first goal is to increase the public appreciation of how chemistry can meet some of the global challenges we face today. This surely goes hand-in-hand with improving the public understanding of chemistry - it is much easier to appreciate the contributions chemistry can make when you have a clear idea of what chemistry actually is. Making headway in this area is not going to be easy, especially as chemistry generally has a bad press and is often misunderstood in the mainstream media. The 'chemicals are bad' mantra is hard to overcome ${ }^{5}$.

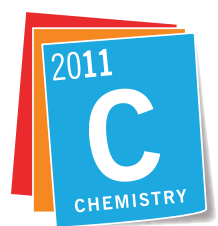

\section{International Year of CHEMISTRY 2011}

Two other stated goals of the IYC are to get young people more interested in chemistry and generate more enthusiasm for its creative aspects. Both of these aims are obviously tied up with the future of our subject - chemistry is far from finished as an intellectual discipline (despite what may be written about it elsewhere ${ }^{6}$ ). There are undoubtedly many discoveries yet to be made and new generations of enquiring young minds will be required to make them.

Two important areas of chemical research highlighted by the IYC prospectus are molecular medicine and advanced materials. Developments in these areas are crucial to modern life and yet they are not always recognized as being fundamentally chemical at their core. Here is an opportunity for chemists to clearly articulate to a wider audience just how much of contemporary science, medicine and technology is underpinned by chemistry. Take materials as just one example; we need to get the message across that many of the materials intrinsic to our everyday lives would not exist without advances in chemistry - consider how many synthetic organic polymers or designer inorganic ceramics you rely on in a typical day, and imagine what life would be like without them.

Because chemistry has such a broad reach and touches on so many other disciplines it is often described as the central science. The range of topics for which the Nobel Prize in Chemistry has been awarded in recent years is perhaps a testament to the diversity of chemical research, although this has also prompted some debate ${ }^{7}$ as to what is - and is not - chemistry. The danger is that chemistry becomes so diffuse across many different areas of science that it loses some of its identity in its own right. Chemists must make sure that chemistry comes across loud and clear, and takes credit where credit is due. It's time to remind everyone that, for example, many of the cutting-edge forensic techniques we regularly see played out on our TV screens are built on fundamental chemical principles. And that DNA sequencing - which enables us to figure out the genomes of an everincreasing variety of species - would be awfully difficult were it not for advances in chemistry.

The IYC provides a focal point for the past successes of our subject to be celebrated and its future potential to be emphasized - but we should be wary of simply preaching to the choir. As chemists, we have a pretty good grasp of how important our subject is and where it sits in the grand scheme of science itself. The biggest impact of the IYC should not be on chemists, but those who are not (or not yet) chemists. The IYC provides a framework through which chemistry should be clearly and enthusiastically communicated with the wider public, highlighting how crucial it is in everyday life and why it is vital for our future.

\footnotetext{
References

1. http://www.un.org/observances/years.shtml

2. http://go.nature.com/odKdMY

3. http://www.chemistry2011.org/

4. http://go.nature.com/gTliTV

5. Nature Chem. 2, 599 (2010).

6. http://go.nature.com/9MwI2P

7ature Chem. 1, 671 (2009).
} 